\title{
c-MYC-induced long noncoding RNA MEG3 aggravates kidney ischemia-reperfusion injury through activating mitophagy by upregulation of RTKN to trigger the Wnt/ $\beta$-catenin pathway
}

\author{
Dajun Liư', Ying Liư ${ }^{1}$ Xiaotong Zheng ${ }^{1}$ and Naiquan Liu ${ }^{1}$
}

\begin{abstract}
Ischemia-reperfusion injury (IRI)-induced acute kidney injury (AKI) is a life-threatening disease. The activation of mitophagy was previously identified to play an important role in IRI. Maternally expressed 3 (MEG3) can promote cerebral IRI and hepatic IRI. The present study was designed to study the role of MEG3 in renal IRI. Renal IRI mice models were established, and HK-2 cells were used to construct the in vitro models of IRI. Hematoxylin-eosin staining assay was applied to reveal IRI-triggered tubular injury. MitoTracker Green FM staining and an ALP kit were employed for detection of mitophagy. TdT-mediated dUTP-biotin nick-end labeling assay was used to reveal cell apoptosis. The results showed that renal cortex of IRI mice contained higher expression of MEG3 than that of sham mice. MEG3 expression was also elevated in HK-2 cells following IRI, suggesting that MEG3 might participate in the development of IRI. Moreover, downregulation of MEG3 inhibited the apoptosis of HK-2 cells after IRI. Mitophagy was activated by IRI, and the inhibition of MEG3 can restore mitophagy activity in IRI-treated HK-2 cells. Mechanistically, we found that MEG3 can bind with miR-145-5p in IRI-treated cells. In addition, rhotekin (RTKN) was verified to serve as a target of miR145-5p. MEG3 upregulated RTKN expression by binding with miR-145-5p. Further, MEG3 activated the Wnt/ $\beta$-catenin pathway by upregulation of RTKN. The downstream effector of Wnt/B-catenin pathway, c-MYC, served as the transcription factor to activate MEG3. In conclusion, the positive feedback loop of MEG3/miR-145-5p/RTKN/Wnt/ $\beta$-catenin/c-MYC promotes renal IRI by activating mitophagy and inducing apoptosis, which might offer a new insight into the therapeutic methods for renal IRI in the future.
\end{abstract}

\section{Introduction}

Ischemia-reperfusion injury (IRI) is caused by a sudden temporary impairment of the blood flow to the particular organ and can cause a variety of ischemic diseases, including brain stroke, myocardial infarction, and ischemic acute kidney injury $(\mathrm{AKI})^{1}$. Although reperfusion is essential for the survival of ischemic kidney tissues, it can lead to additional damage to the kidney that results in poorer prognosis of AKI patients ${ }^{2,3}$.

Correspondence: Dajun Liu (liudjun263@hotmail.com)

'Department of Nephrology, Shengjing Hospital of China Medical University, 110022 Shenyang, Liaoning, China

Edited by A. Stephanou
AKI is characterized by tubular epithelial cell death and dysfunction at the cellular level ${ }^{4}$. The morbidity and mortality rates of patients with AKI in intensive care unit can reach $50-70 \%{ }^{5}$.

Although the molecular mechanisms underlying apoptosis of renal tubular epithelial cells during IRI remain unclear, numerous studies indicate that mitochondrial dysfunction plays a critical role in apoptosis of tubular epithelial cells ${ }^{6}$. Cellular stresses, including hypoxia, ischemia, and glucose deprivation, can cause damage or dysfunction of mitochondria ${ }^{7}$. In addition, researches showed that mitophagy exerts a protective function in the liver ${ }^{8}$. Mitophagy, a complex process that rapidly and

\section{(c) The Author(s) 2021}

(c) Open Access This article is licensed under a Creative Commons Attribution 4.0 International License, which permits use, sharing, adaptation, distribution and reproduction c. in any medium or format, as long as you give appropriate credit to the original author(s) and the source, provide a link to the Creative Commons license, and indicate if changes were made. The images or other third party material in this article are included in the article's Creative Commons license, unless indicated otherwise in a credit line to the material. If material is not included in the article's Creative Commons license and your intended use is not permitted by statutory regulation or exceeds the permitted use, you will need to obtain permission directly from the copyright holder. To view a copy of this license, visit http://creativecommons.org/licenses/by/4.0/. 
selectively removes long-lived or damaged mitochondria in an autophagy-dependent manner, is an adaptive response of mitochondria to various stress damages, which was previously identified to play an important role in IRI $^{9}$. The most recognized mitophagy pathway in mammalian cells is dependent on Parkin ${ }^{10}$. A study revealed that Parkin-related mitophagy promotes cardiomyocyte death in IRI ${ }^{11}$. Another study has indicated that deficiency of autophagy aggravates tubular injury in proximal tubules and leads to damage of mitochondria in IRI-induced AKI model ${ }^{12}$.

Long noncoding RNAs (lncRNAs) are noncoding RNAs with the length $>200$ nucleotides and lack the protein-coding capacity ${ }^{13}$. LncRNAs are potential biomarkers or therapeutic targets for $\mathrm{AKI}^{14}$. The competing endogenous RNA (ceRNA) pattern refers to that lncRNAs competitively bind with microRNAs (miRNAs) to antagonize the suppressive effects of miRNAs on downstream targets ${ }^{15}$, and lncRNAs were widely reported to serve as ceRNAs in the pathogenesis of AKI. For instance, XIST binds with miR-142-5p to upregulate PDCD4 and thus alleviates AKI ${ }^{16}$. The LINC00343/rnomiR-1956-5p/KCP axis is involved in contrast-induced $\mathrm{AKI}^{17}$. LINC00520 upregulates OSMR expression to promote AKI progression by binding with miR-27b-3p ${ }^{18}$. TCONS_00016233 contributes to sepsis-induced AKI via modulation on the miR-22-3p/AIFM1 axis ${ }^{19}$. Moreover, IncRNAs are crucial mediators for mitophagy. PVT1 is activated during muscle atrophy and impacts mitochondrial respiration, mitophagy, and apoptosis in vivo ${ }^{20}$. RMST enhances FUS SUMOylation to inhibit mitophagy of glioma cells ${ }^{21}$.

Maternally expressed 3 (MEG3) is an imprinted gene with a location at $14 \mathrm{q} 32.2$ and is a significant mediator for IRI. MEG3 facilitates cerebral IRI by increasing pyroptosis via the miR-485/AIM2 axis $^{22}$ and the $\mathrm{Wnt} / \beta$-catenin pathway $^{23}$. MEG3 contributes to hepatic IRI via the miR$34 \mathrm{a} / \mathrm{Nrf2}$ signaling pathway ${ }^{24}$. Moreover, MEG3 promotes myocardial IRI by downregulation of miR-7-5 $\mathrm{p}^{25}$. MEG3 functions as a ceRNA against miR-21 to regulate ischemic neuronal death ${ }^{26}$. MEG3 inhibits cancer cell proliferation and acts as an antioncogene in gastric cancer ${ }^{27}$, prostate cancer $^{28}$, and laryngeal cancer ${ }^{29}$. The functions and underlying mechanisms of MEG3 in the progression of kidney IRI remain to be further explored. We hypothesized that MEG3 can promote kidney IRI via the ceRNA pattern. In the present study, human kidney proximal tubular cells (HK-2) were used to establish an IRI model in vitro. Kidney IRI mice were used as the in vivo models. MEG3 was knocked down in IRI mice and in HK-2 cells, and loss-of-function assays revealed the effects of MEG3 on renal function and on cell apoptosis and mitochondrial dysfunction during IRI.

\section{Results}

\section{MEG3 exhibited high expression after IRI}

To identify the role of MEG3 in kidney IRI, we constructed IRI mice models. Results of hematoxylin-eosin $(H \& E)$ staining assay revealed that IRI triggered tubular injury (Fig. 1A). Moreover, a TdT-mediated dUTP-biotin nick-end labeling (TUNEL) assay revealed that IRI induced more apoptosis in kidney tissues of mice (Fig. 1B). These data illustrated that the IRI models were successfully established. Then reverse transcription quantitative real-time PCR (RT-qPCR) was conducted to evaluate the expression of MEG3 in renal cortex. The results revealed that MEG3 exhibited higher expression post IRI in renal cortex compared with that in the sham group (Fig. 1C). Similarly, the expression of MEG3 was elevated in HK-2 cells $6 \mathrm{~h}$ following IRI (Fig. 1D).

\section{Suppression of MEG3 inhibited apoptosis and mitophagy in HK-2 cells following IRI}

Furthermore, we intended to identify whether MEG3 is involved in the pathogenesis of IRI. MEG3 was knocked down in HK-2 cells by transfection of sh-MEG3. RTqPCR analysis showed that IRI resulted in a significant increase of the expression of MEG3, and transfection of sh-MEG3 caused a significant decrease of the expression of MEG3 in HK-2 cells (Fig. 2A). Afterwards, activity of the caspase-3 (cell apoptosis index) was measured via a caspase-3 detection kit. The results showed that IRI enhanced the activity of caspase- 3 , and this effect was rescued by downregulation of MEG3 (Fig. 2B). Moreover, the increased number of TUNEL-positive cells caused by IRI was inversely changed by deficiency of MEG3 in HK-2 cells (Fig. 2C, D). These data demonstrated that downregulation of MEG3 inhibited the apoptosis of HK-2 cells after IRI. Thereafter, whether MEG3 is involved in mitophagy in HK-2 cells was explored. Protein levels of Parkin, LC3B-I, LC3B-II (an autophagosome marker), and p62 were detected. p62, a mitophagy adaptor, can translocate to damaged mitochondria and be digested during mitophagy, and the decrease of p62 indicates mitophagy ${ }^{30}$. Results from western blot analysis indicated that the protein levels of Parkin, LC3B-I, and LC3B-II were increased and the protein levels of p62 were reduced following IRI, while silencing of MEG3 rescued these effects (Fig. 2E). Subsequently, mitophagy flux was detected by measurement on the changes of mitochondria repressed by Baf (lysosome function inhibitor). As shown in Fig. 2F, G, mitophagy flux was significantly enhanced following IRI and was further reduced by silenced MEG3. In addition, ATP production was inhibited in response to IRI, while downregulation of MEG3 effectively neutralized this effect (Fig. 2H). 
A

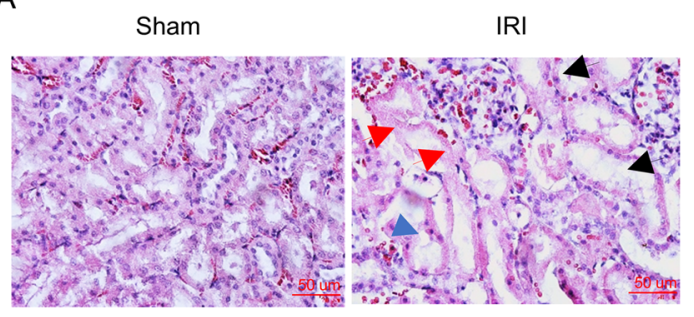

C

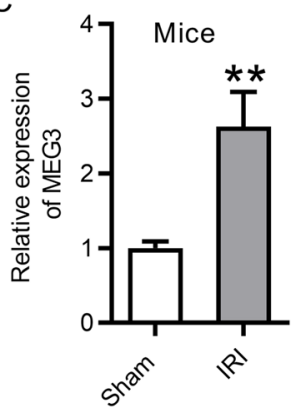

D

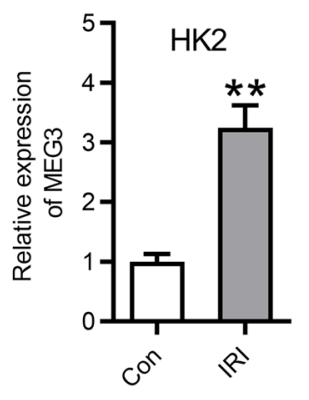

B

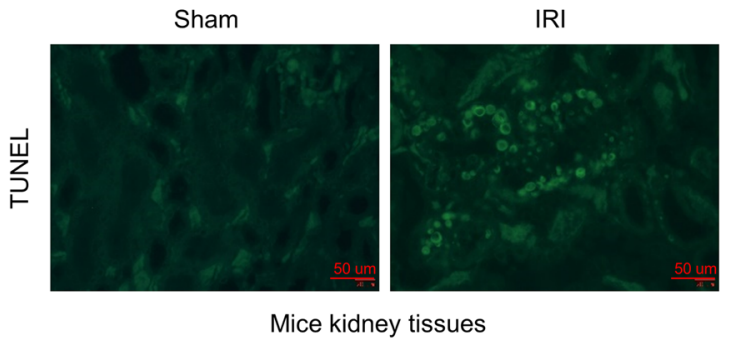

Fig. 1 MEG3 exhibited high expression after IRI. A H\&E staining revealed IR triggered tubular injury. B TUNEL assay revealed the apoptosis cells induced by IRI in mice kidney tissues. Red arrow indicates coagulative necrosis with tubular structures; black arrow indicates renal tubule vacuole; blue arrow indicates renal tubular necrosis and detachment. C RT-qPCR revealed expression of MEG3 over a 12-h post IRI in renal cortex of mice compared with that in the sham group. D RT-qPCR revealed the expression of MEG3 in HK-2 cells $6 \mathrm{~h}$ following IRI. ${ }^{* *} p<0.01$.

\section{Silencing of MEG3 attenuated the kidney injury in vivo}

Next, the effects of MEG3 suppression on kidney injury in vivo were investigated. RT-qPCR indicated that injection of AAV-sh-MEG3 vector led to a significant decrease of the expression of MEG3 in the kidney of mice (Fig. 3A). $H \& E$ staining assay exhibited that IR-triggered tubular injury was alleviated by downregulation of MEG3 (Fig. 3B). The increased number of TUNEL-positive apoptotic cells induced by IRI was decreased by MEG3 inhibition (Fig. 3C, D). Silencing of MEG3 rescued the increase of caspase- 3 activity in renal tissues of IRI mice (Fig. 3E). Additionally, IR induced elevation of the levels of blood urea nitrogen (BUN) and creatinine in the serum were rescued by the suppression of MEG3 (Fig. 3F, G). The number of damaged tubules was reduced by the inhibition of MEG3 following IRI (Fig. 3H). Moreover, IRI induced mitophagy, and silencing of MEG3 inhibited mitophagy activity (Fig. 3I-K).

MEG3 can bind with miR-145-5p in HK-2 cells following IR

Afterwards, the underlying ceRNA mechanism of MEG3 involved in IRI was investigated. StarBase database (http://starbase.sysu.edu.cn/) was used to identify the potential miRNAs binding with MEG3 (screening condition: strict stringency $(\geq 5)$ in CLIP data). According to the data, we found that there are five candidate miRNAs (miR-145-5p, miR-5195-3p, miR-3150-3p, miR-6763-5p,
miR-4640-3p) that might bind with MEG3. Among them, miR-145-5p and miR-3150-3p were decreased in IRtreated HK-2 cells compared with that in the control group (Fig. 4A). The following assays were conducted in IR-treated HK-2 cells. Downregulation of MEG3 resulted in the increase of miR-145-5p expression and had no significant effect on miR-3150-3p in HK-2 cells (Fig. 4B). Thus miR-145-5p was applied for further molecular exploration. RNA immunoprecipitation (RIP) assay was subsequently conducted, and the results revealed that MEG3 and miR-145-5p were enriched in the Ago2 group rather than in the IgG group, indicating the coexistence of MEG3 and miR-145-5p in RNA-induced silencing complexes (Fig. 4C). Further, miR-145-5p was effectively overexpressed or knocked down in HK-2 cells by transfection of miR-145-5p mimics or miR-145-5p inhibitor with NC mimics or NC inhibitor as respective controls. Based on the data from RT-qPCR, the expression of miR145-5p was increased by miR-145-5p mimics and was decreased by miR-145-5p inhibitor in IR-treated HK-2 cells (Fig. 4D). The binding sequences of miR-145-5p and MEG3 were predicted from starBase. We mutated the binding sequences of MEG3 on miR-145-5p to explore whether they are responsible for the binding between MEG3 and miR-145-5p (Fig. 4E). Luciferase reporter assay revealed that the luciferase activity of MEG3-WT was significantly reduced by miR-145-5p mimics and 


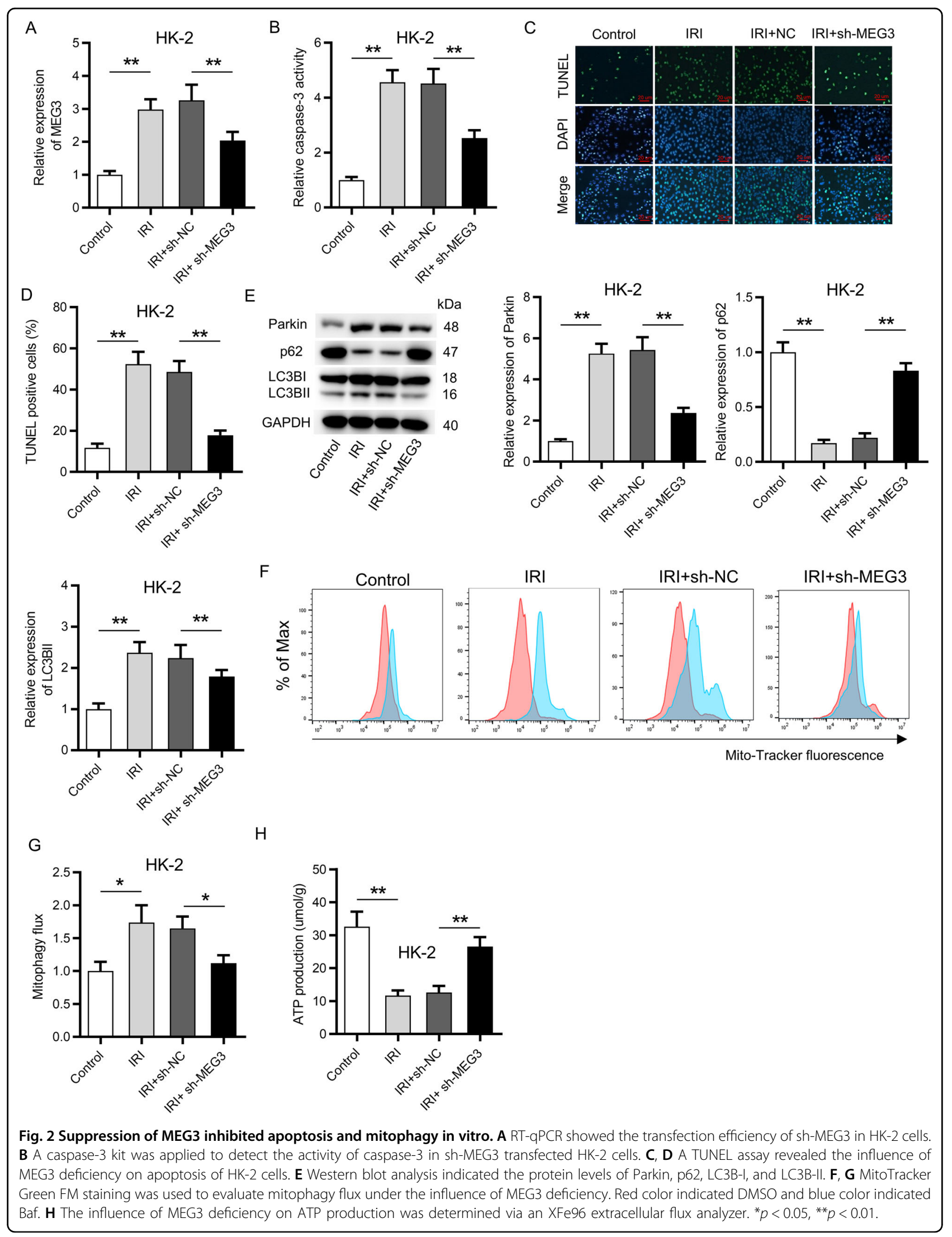




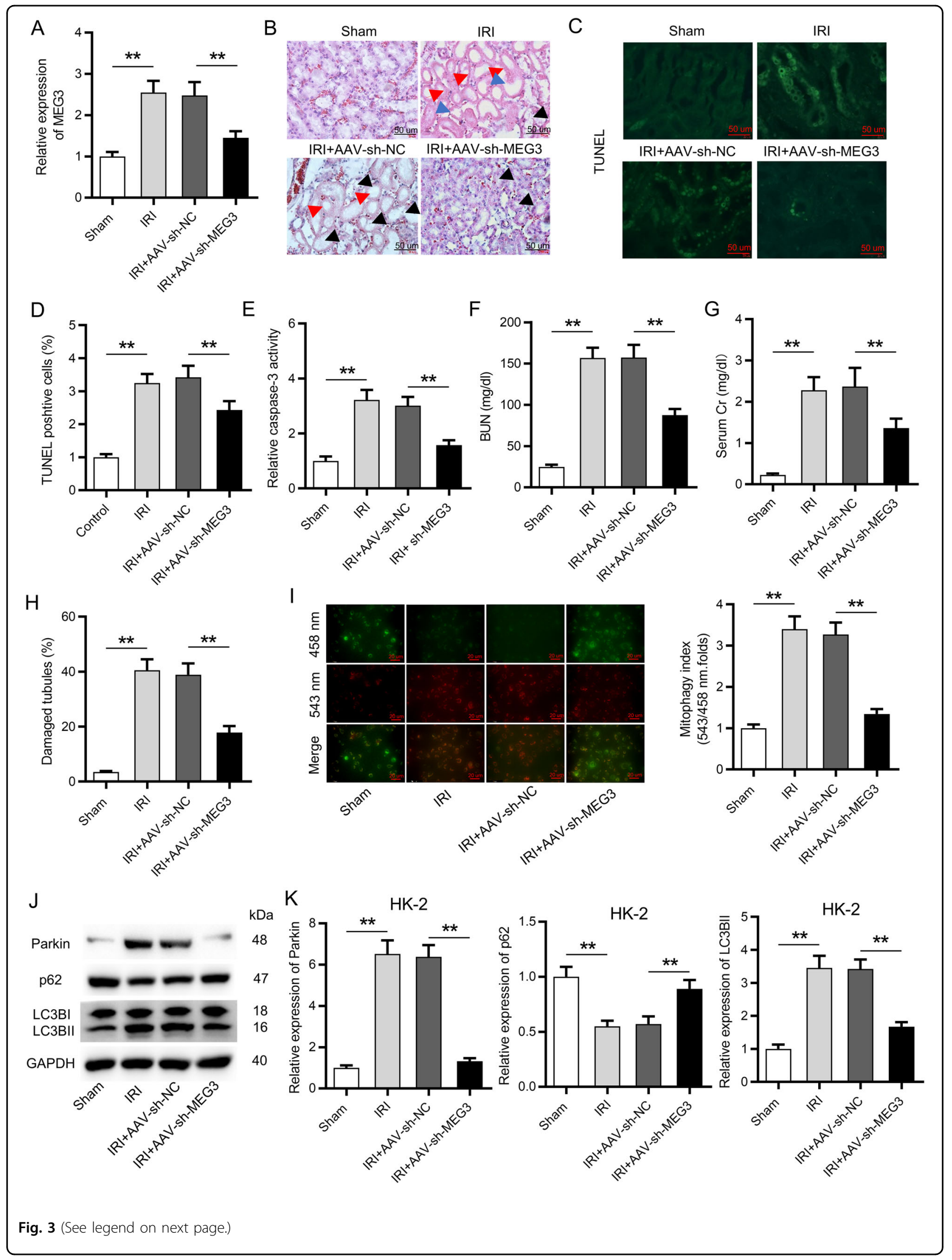


(see figure on previous page)

Fig. 3 Silencing of MEG3 attenuated the kidney injury in vivo. A RT-qPCR indicated the expression of MEG3 in the kidney of mice after injection of AAV-sh-MEG3 vector. $\mathbf{B}$ H\&E staining exhibited the effects of downregulation of MEG3 on tubular injury. Red arrow indicates coagulative necrosis with tubular structures; black arrow indicates renal tubule vacuole; blue arrow indicates renal tubular necrosis and detachment. C, D A TUNEL assay was applied to detect the number of TUNEL-positive cells. $\mathbf{E}$ Influence of silenced MEG3 on caspase 3 activity. F, G The effects of silenced MEG3 on the levels of BUN and creatinine in the serum were measured. $\mathbf{H}$ The effects of silenced MEG3 on tubular injury index were determined. I An mtKeima assay was used to detect the mitophagy activity following IRI and under the influence of MEG3 deficiency. J, K Western blot analysis of Parkin, p62, LC3B-I, and LC3B-II levels in kidney tissues from IRI mice and sham mice. ${ }^{* *} p<0.01$.

enhanced by miR-145-5p inhibitor, while the luciferase activity of MEG3-Mut presented no significant change in both groups (Fig. 4F).

\section{RTKN served as a target of miR-145-5p}

Then the downstream targets of miR-145-5p were probed. As revealed in Fig. 5A, there are three potential targets (PCBP2, RTKN, and RAD23B) of miR-145-5p (screening condition: strict stringency $(\geq 5)$ in CLIP data; high stringency $(\geq 3)$ in degradome data; program number: 5 programs). RTKN expression was higher in HK-2 cells post IRI than in control cells, while the expression of PCBP2 and RAD23B exhibited no significant changes (Fig. 5B). Moreover, the mRNA expression of RTKN was increased by miR-145-5p inhibitor and was reduced by upregulation of miR-145-5p (Fig. 5C). Additionally, RIP assay demonstrated that miR-145-5p and RTKN were enriched in Ago2 group instead of IgG group (Fig. 5D), indicating that miR-145-5p and RTKN coexisted in RNA induced silenced complexes. Moreover, the predicted binding sites of miR-145-5p and RTKN were obtained from starBase (Fig. 5E). The upregulation of miR-145-5p decreased the luciferase activity of RTKN-WT, and downregulation of miR-145-5p increased the luciferase activity of RTKN-WT. However, both the transfection of miR-145-5p mimics and the miR-145-5p inhibitor exhibited no significant effects on the luciferase activity of RTKN-Mut, suggesting that miR-145-5p targeted RTKN at the predicted sites (Fig. 5F, G). Furthermore, silencing of MEG3 reduced the expression of RTKN, while inhibition of miR-145-5p rescued the suppressive effects of silenced MEG3 on RTKN expression, indicating that MEG3 upregulated RTKN expression via binding with miR-145-5p (Fig. 5H).

\section{Inhibition of RTKN reduced cell apoptosis and mitophagy in HK-2 cells followed by IRI}

Furthermore, the functions of RTKN on HK-2 cells following IRI were probed. We first silenced the expression of RTKN via transfection of sh-RTKN. The effective knockdown efficiency of RTKN was validated by RTqPCR analysis (Fig. 6A). TUNEL assay depicted that silencing of RTKN rescued the promoting effect of IR treatment on cell apoptosis (Fig. 6B, C). The following function assays revealed that inhibition of RTKN partially restored mitophagy activity induced by IRI in HK-2 cells (Fig. 6D-F).

\section{Upregulation of RTKN rescued the effects of silenced MEG3 on cell apoptosis and mitophagy}

Subsequently, rescue assays were performed in IRtreated HK-2 cells. Suppression of MEG3 decreased the caspase- 3 activity and RTKN overexpression rescued this effect (Fig. 7A). The number of TUNEL-positive cells was reduced in response to downregulation of MEG3 and was elevated by upregulation of RTKN (Fig. 7B, C). RTKN overexpression rescued the effects of inhibited MEG3 on the protein levels of Parkin, p62, LC3B-I, and LC3B-II (Fig. 7D). In addition, upregulation of RTKN rescued the inhibitory effects of silenced MEG3 on the mitophagy activity in IRI-treated HK-2 cells (Fig. 7E, F). In addition, increased ATP production caused by MEG3 knockdown was rescued by the overexpression of RTKN (Fig. 7G).

\section{c-MYC, the downstream effector of the Wnt/ $\beta$-catenin pathway, promoted the transcription of MEG3}

The Wnt/ $\beta$-catenin pathway is activated in the pathogenesis of AKI. Both MEG3 and RTKN were reported to trigger the Wnt/ $\beta$-catenin pathway. Thus we conducted the FOP/TOP luciferase reporter assay to detect the influence of MEG3 and RTKN on the $\mathrm{Wnt} / \beta$-catenin pathway in IR-treated HK-2 cells. As revealed in Fig. 8A, the Wnt/ $\beta$-catenin pathway was activated by IR treatment in HK-2 cells. Silencing of MEG3 or RTKN reduced the TOP activity, and upregulation of RTKN rescued the inhibitory effects of silenced MEG3 on TOP activity, indicating that MEG3 can activate the $\mathrm{Wnt} / \beta$-catenin pathway by upregulation of RTKN. Moreover, protein expression of $\beta$-catenin and c-MYC was reduced by silenced MEG3 or RTKN, and RTKN rescued the inhibitory effects of silenced MEG3 on $\beta$-catenin and c-MYC protein levels (Fig. 8B). Furthermore, we identified that c-MYC is predicted to regulate MEG3 ${ }^{31}$ and c-MYC commonly serves as a transcription factor. Whether transcription of MEG3 is activated by c-MYC was subsequently explored. mRNA and protein expression of c-MYC were reduced by transfection of sh-c-MYC\#1/2 in IRI-treated HK-2 cells (Fig. 8C). Knockdown of c-MYC significantly reduced the expression of MEG3 (Fig. 8D). ChIP assay revealed that promoter of MEG3 was 


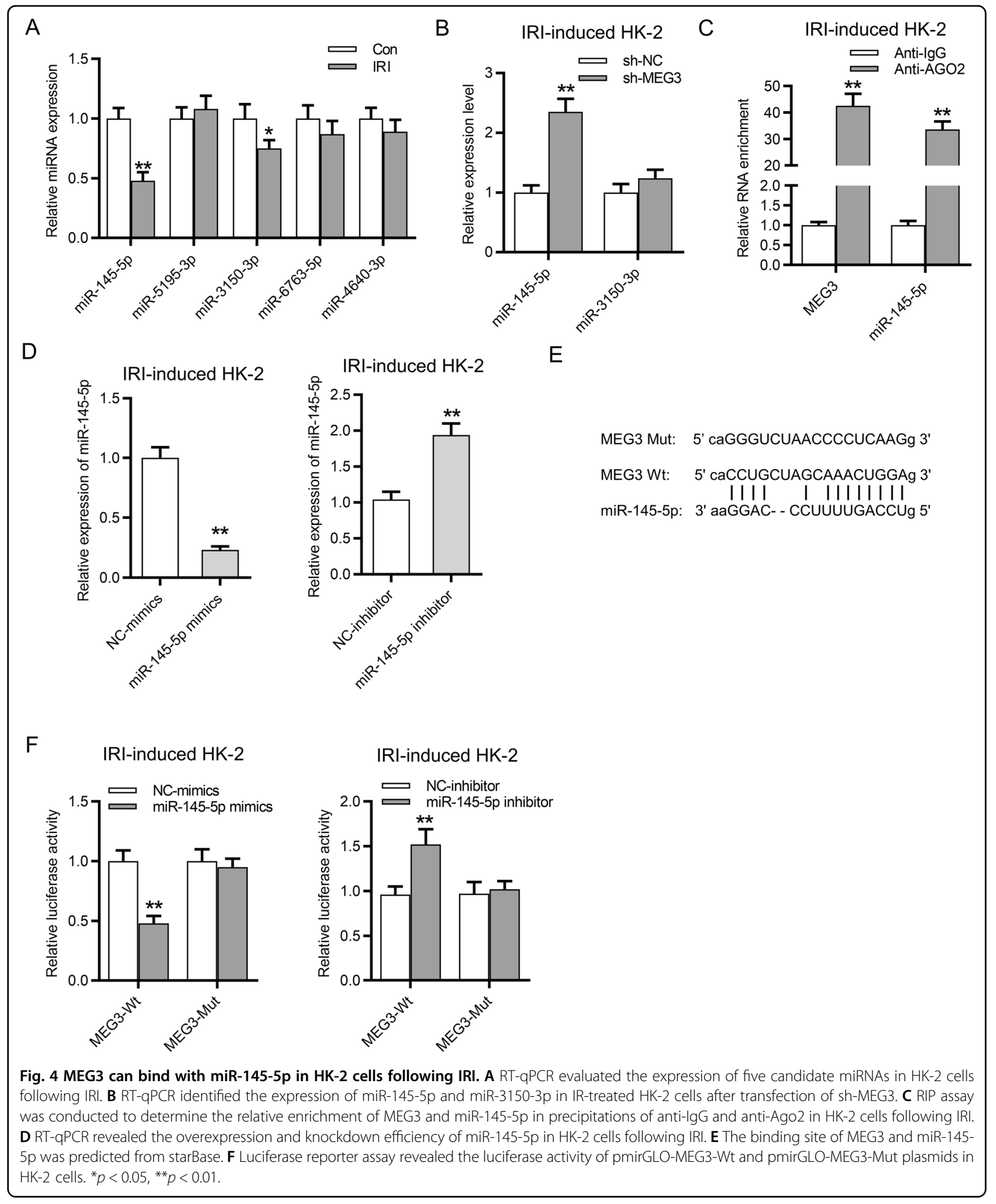

significantly precipitated by anti-c-MYC compared to anti-IgG (Fig. 8E). Finally, the luciferase reporter assay revealed that downregulation of c-MYC significantly reduced the luciferase activity of MEG3 promoter in IRtreated HK-2 cells (Fig. 8F). All these findings revealed that MEG3 activated the Wnt/ $\beta$-catenin pathway by 


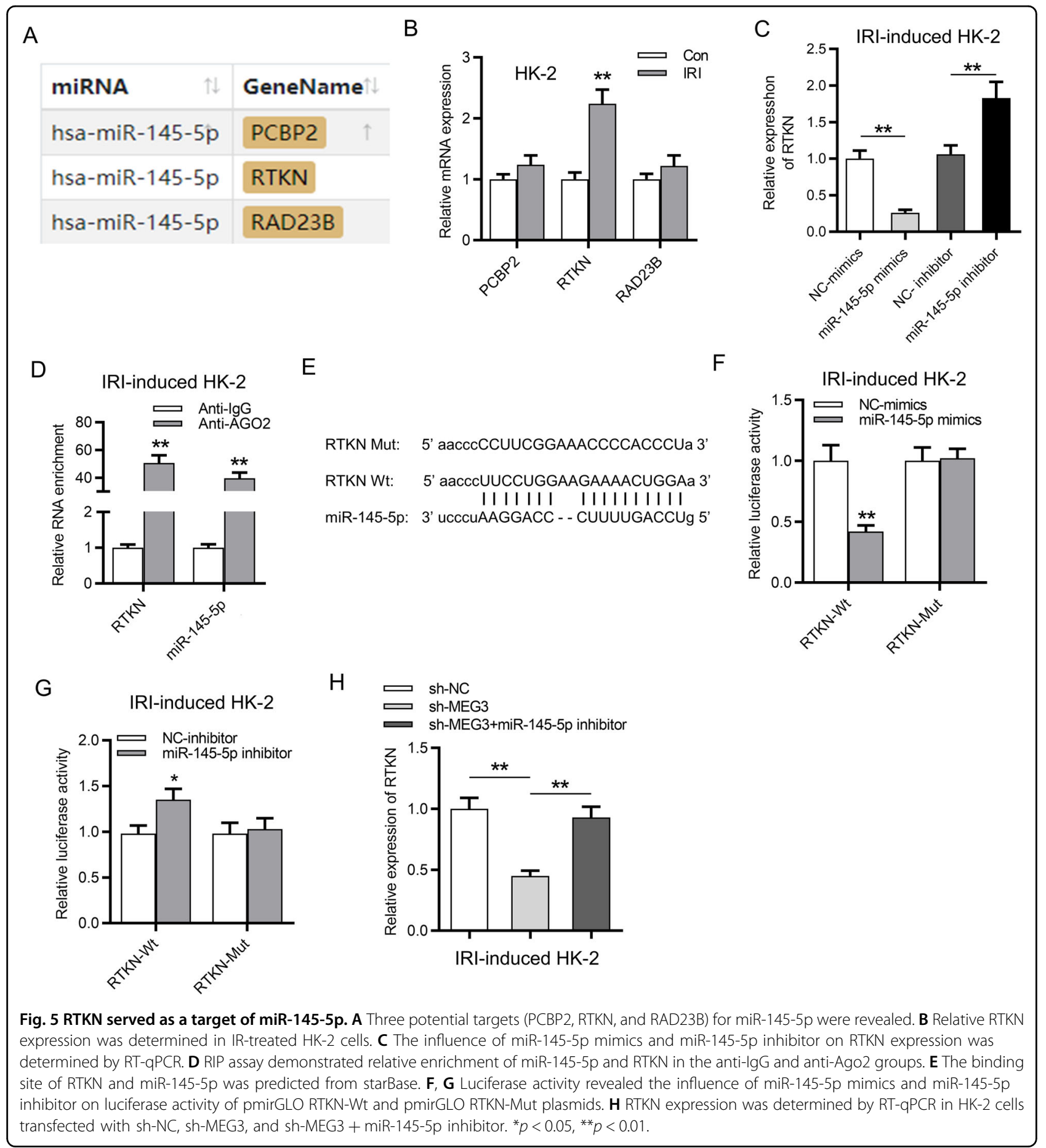

RTKN, and the downstream effector of the Wnt/ $\beta$-catenin pathway, c-MYC, further promoted the transcription of MEG3 in HK-2 cells following IRI.

\section{Discussion}

In the present study, MEG3 was upregulated in in vivo and in vitro models of IRI. Downregulation of MEG3 inhibited the apoptosis and mitophagy of HK-2 cells after
IRI. As a cytoplasmatic lncRNA, MEG3 competitively bound with miR-145-5p to upregulate RTKN and thus activated the $\mathrm{Wnt} / \beta$-catenin pathway. c-MYC, the downstream effector of the $\mathrm{Wnt} / \beta$-catenin pathway, further activated MEG3 at the transcription level.

We first revealed the promoting effects of MEG3 on apoptosis and mitophagy of HK-2 cells post IRI. Another study has indicated that MEG3 inhibits autophagy to 


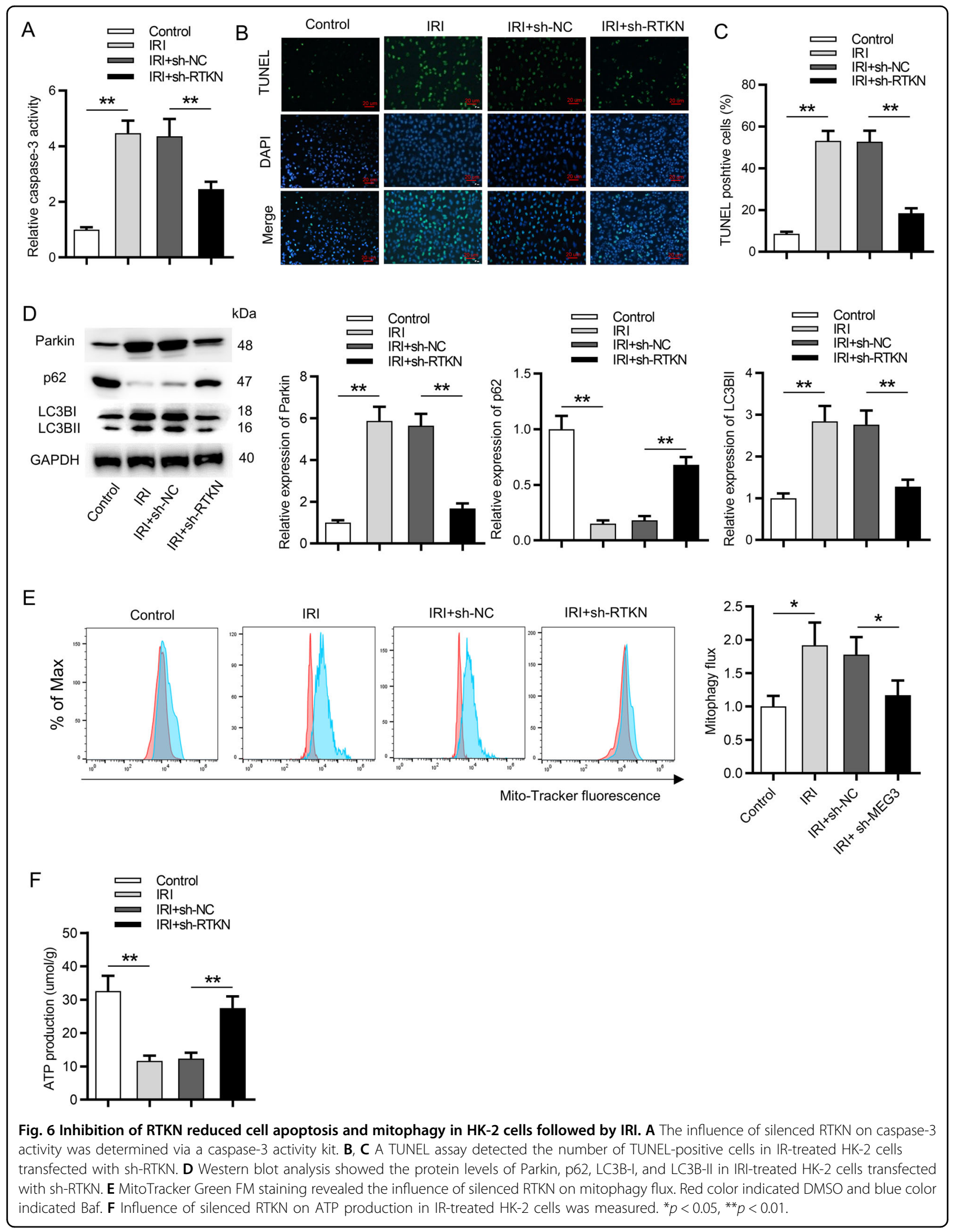




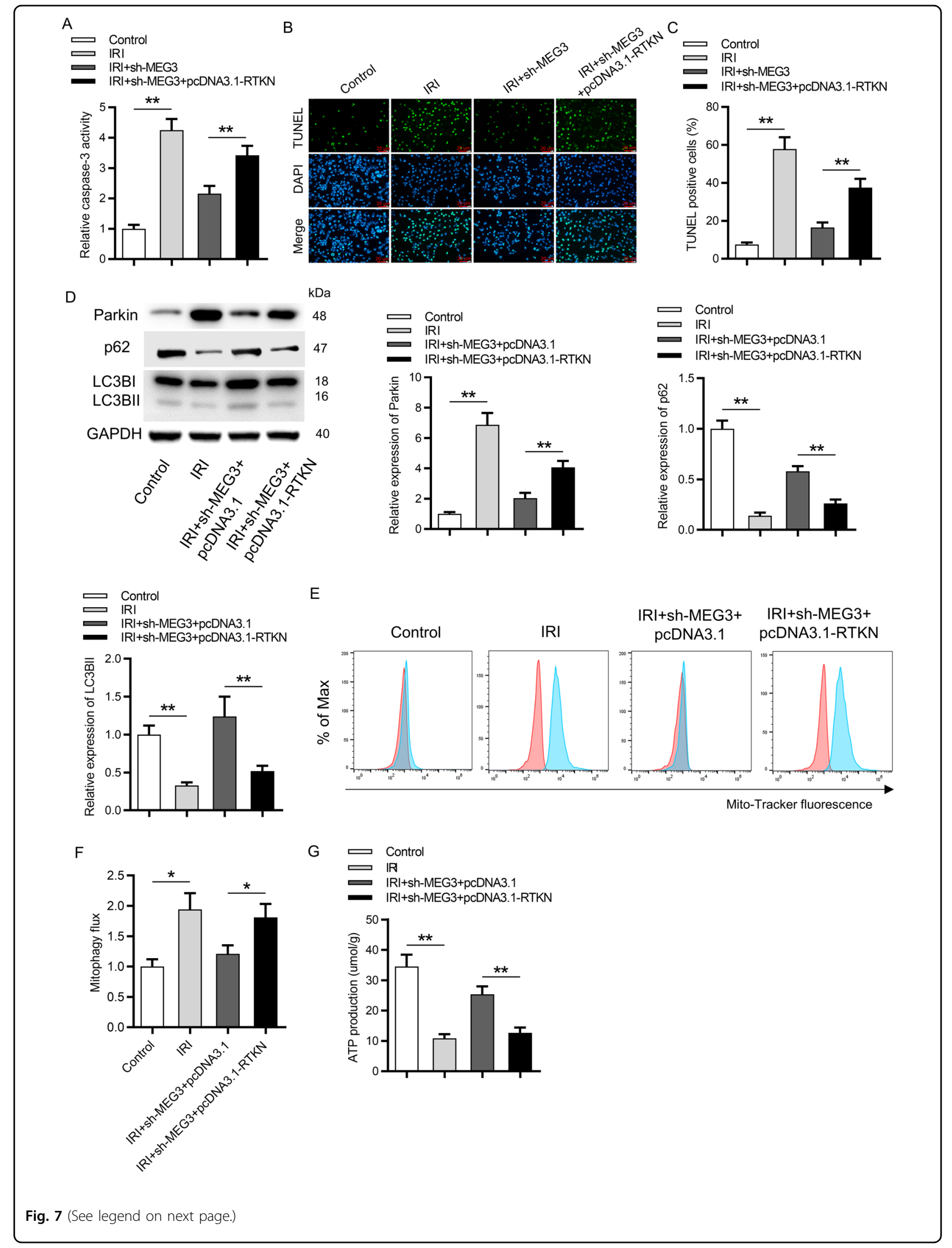


(see figure on previous page)

Fig. 7 Upregulation of RTKN rescued the effect of silencing of MEG3 on cell apoptosis and mitophagy. A The rescue effect of upregulated RTKN on silenced MEG3 in caspase-3 activity was evaluated via a caspase-3 activity kit. B, C TUNEL assay revealed the rescue effect of upregulated RTKN on silenced MEG3 in the number of TUNEL-positive cells. D Western blot analysis showed the protein levels of Parkin, p62, LC3B-I, and LC3B-II in IR-treated HK-2 cells transfected with sh-MEG3 + pcDNA3.1-RTKN. E, F Mitophagy flux was assessed in IR-treated HK-2 cells after cotransfection with sh-MEG3 + pcDNA3.1-RTKN. Red color indicated DMSO and blue color indicated Baf. G ATP production in IRI-treated HK-2 cells cotransfected with sh-MEG3 + pcDNA3.1-RTKN was measured. ${ }^{*} p<0.05,{ }^{* *} p<0.01$.

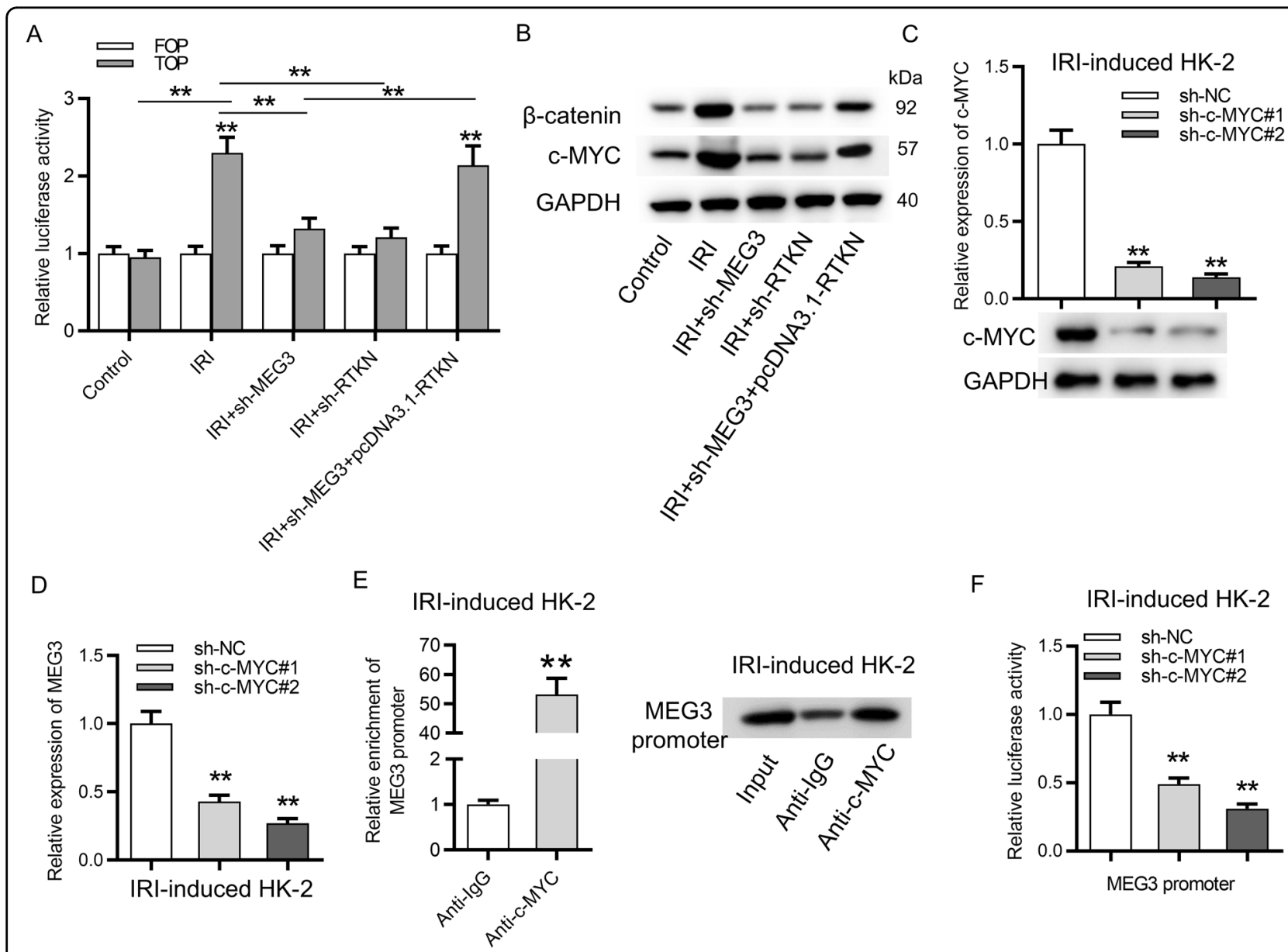

Fig. 8 c-MYC, the downstream effector for the Wnt/ $\beta$-catenin pathway, activated the transcription of MEG3. A FOP/TOP luciferase reporter assay revealed the influence of MEG3 and RTKN on the Wnt/ $\beta$-catenin pathway in IR-treated HK-2 cells. B Western blot analysis of $\beta$-catenin and cMYC proteins. C Knockdown efficiency of c-MYC was verified by RT-qPCR and western blot analyses. D The influence of silenced c-MYC on MEG3 expression was examined by RT-qPCR. E A ChIP assay was employed to detect relative enrichment of MEG3 promoter precipitated by anti-c-MYC. F A luciferase reporter assay was employed to detect the luciferase activity of MEG3 promoter in cells transfected with sh-c-MYC\#1/2. ${ }^{* *} p<0.01$.

contribute to adenosine-induced cytotoxicity in hepatoma HepG2 cells $^{32}$. MEG3 induces autophagy by upregulation of sirtuin 7 and inhibition on the PI3K/AKT/mTOR pathway in glioma cells ${ }^{33}$. MEG3 binds with miR-378 to inhibit the protective influence of miR-378 against neuronal autophagy and neurological functional impairment in ischemic stroke ${ }^{34}$. To explore the underlying ceRNA mechanism of MEG3 in HK-2 cells following IRI, the downstream miRNAs for MEG3 were investigated. MiR- 145-5p was verified to bind with MEG3 and was negatively regulated by MEG3. It has been reported that activation of $\delta$-opioid receptor modifies hypoxia-induced changes in expression of miR-145-5p in the liver ${ }^{35}$. MiR145-5p induces the apoptosis of cardiomyocytes by DUSP6 via the c-Jun N-terminal kinase pathways ${ }^{36}$. Inhibition of miR-145-5p promotes neurological outcomes of rats post middle cerebral artery occlusion and reperfusion via the Nurr1-TNF- $\alpha$ signaling ${ }^{37}$. MiR-145-5p 
alleviates high glucose-induced apoptosis by regulating the Notch signaling pathway in podocytes ${ }^{38}$. In the current study, miR-145-5p expression was downregulated in IRI mice model and in HK-2 cells post IRI.

Subsequently, RTKN, a Rho-guanosine triphosphatase effector, was validated as the target of miR-145-5p. MiR145-5p targeted the 3'-untranslated region of RTKN to inhibit the expression of RTKN. MEG3 can upregulate the expression of RTKN by competitively binding with miR145-5p. The reports on role of RTKN in IRI are limited, and some studies have revealed the oncogenic functions of RTKN to inhibit cell apoptosis in colon cancer ${ }^{39}$, hepatocellular carcinoma ${ }^{40}$, lung cancer ${ }^{41}$, and breast cancer $^{42}$. In the present study, we innovatively demonstrated that RTKN promoted apoptosis and mitophagy in HK-2 cells post IRI. Rescue assays further revealed that upregulation of RTKN rescued the effects of silenced MEG3 on apoptosis and mitophagy activity in IR-treated HK-2 cells.

The Wnt/ $\beta$-catenin pathway is activated in $\mathrm{AKI}^{43,44}$ and can promote apoptosis of HK-2 cells ${ }^{45,46}$. The Wnt/ $\beta$-catenin pathway can activate autophagy ${ }^{47-49}$ or be triggered by autophagy ${ }^{50}$. Moreover, MEG3 downregulates miR-183 to activate BRI3 and thus triggers the Wnt/ $\beta$-Catenin pathway in BON1 cells ${ }^{51}$. MEG3 aggravates the hypoxia injury by Sox 2 to activate the Wnt/ $\beta$-catenin pathway in PC12 cells in a miR-147-dependent manner ${ }^{52}$. Additionally, RTKN2 has been indicated to activate the $\mathrm{Wnt} / \beta$-catenin signaling pathway ${ }^{53}$. Our findings revealed that MEG3 can activate the Wnt/ $\beta$-Catenin pathway by positive modulation on RTKN in a miR-145-5p-dependent manner. The pro-apoptotic functions of MEG3 on HK-2 cells might be dependent on the Wnt/ $\beta$-Catenin pathway. c-MYC belong to the MYC family, is the downstream effector of the Wnt/ $\beta$-catenin pathway, and was reported to regulate MEG3 expression $^{31}$. In our study, we identified that c-MYC served as the transcription factor for MEG3 to activate upregulation of MEG3. Xiang et al. have revealed that MYC/c-MYC-induced cell proliferation serves as the main factor for mitochondrial decrease caused by mitophagy $^{54}$. Xiong et al. have indicated that MYC impedes mitophagy-dependent necroptosis in diffuse large B cell lymphoma ${ }^{55}$. Another family member of MYC, n-MYC, blocks mitophagy and confers protection to reactive oxygen species in mitochondria via upregulation of TRAP1 in neuroblastoma ${ }^{56}$.

In conclusion, our study revealed that c-MYCactivated MEG3 aggravates IR-induced AKI through activating mitophagy and promoting apoptosis by upregulation of RTKN in a miR-145-5p-dependent way to trigger the $\mathrm{Wnt} / \beta$-Catenin pathway, which might offer a new insight into the therapeutic methods for IR-induced AKI in the future.

\section{Materials and methods \\ Ethics statement}

The experimental protocol and animal use plan in this study were approved by the Animal Ethics Committee of Shengjing Hospital of China Medical University (Liaoning, China).

\section{IRI mice model}

The kidney IRI mice models were established according to a previous study ${ }^{57}$. Eight-week-old adult C57BL/6 mice of both sexes weighing 22-25 g ( $n=6-8$ /group) were obtained from Vital river (Beijing, China). Briefly, pentobarbital sodium was used for anesthetizing the mice by intraperitoneal injection $(50 \mathrm{mg} / \mathrm{kg})$. Post removing the hair, all mice were maintained on thermostatic blanket with a rectal probe to keep the body temperature at $36.5^{\circ} \mathrm{C}$ for surgery. The kidney pedicles were exposed and the flank incisions for bilateral clamping were conducted to block blood supply of kidney for inducing ischemia for $30 \mathrm{~min}$ followed by $12 \mathrm{~h}$ of reperfusion. Mice in the sham group received the same operation except clamping. Animals were grouped using a method of randomization. AAV-sh-MEG3 vector (serotype 2) was delivered (at the titer of $2.0 \times 10^{11} \mathrm{v} . \mathrm{g} / \mathrm{mL}$ ) into mice via tail vein. The mice were sacrificed 28 days after AAV delivery. Finally, the kidneys were dissected, and blood was collected for biochemical assays.

\section{Cell culture and transfection}

HK-2 cells (catalog number: CRL-2190) were obtained from American Type Culture Collection (ATCC) and were cultured in Dulbecco's modified Eagle's medium supplemented with $10 \%$ fetal bovine serum (Invitrogen, Carlsbad, CA, USA) at $37^{\circ} \mathrm{C}$ with $5 \% \mathrm{CO}_{2}$ in a humidified atmosphere. Short hairpin RNA against MEG3 (shMEG3), miR-145-5p mimics/inhibitor, pcDNA3.1-RTKN vector, and respective controls (sh-NC, NC mimics/inhibitor, pcDNA3.1-NC) were constructed by GenePharma (Shanghai, China) and transfected into HK-2 cells using the Lipofectamine 2000 reagent (Invitrogen). Sequence for sh-MEG3 is GGACACATGAACGACTGAATT; sequence for miR-145-5p mimics is GUCCAGUUUUCC CAGGAAUCCCU; sequence for miR-145-5p inhibitor is AGGGAUUCCUGGGAAAACUGGAC; sequence for sh-c-MYC\#1 is CAGTTGAAACACAAACTTGAA; and sequence for sh-c-MYC\#2 is CCTGAGACAGATCAG CAACAA. At transfection for $48 \mathrm{~h}$, cells were harvested for subsequent analyses.

\section{IRI cell model}

HK-2 cells were used to construct the IRI cell model and were cultured in complete renal epithelial cell growth media with $95 \%$ air and $5 \% \mathrm{CO}_{2}$ at $37^{\circ} \mathrm{C}$. HK-2 cells were seeded onto a six-well plate. After $24 \mathrm{~h}$ of incubation, 
HK-2 cells were cultured with hypoxia treatment at $0.5 \%$ $\mathrm{O}_{2}$ for $15 \mathrm{~h}$ and were further cultured in normal culture medium with constant oxygen for $6 \mathrm{~h}$.

\section{Determination of mitophagy}

Mt-Keima is a fluorescent probe targeting the mitochondrial matrix sensitive to $\mathrm{pH}$. The ratio of mt-Keimaderived fluorescence $(543 / 458 \mathrm{~nm})$ was calculated as the value of mitophagy. Low ratio mt-Keima emission (543/ $458 \mathrm{~nm}$ ) reports a neutral environment, while high ratio fluorescence reports an acidic $\mathrm{pH}$. Thus the difference between mitochondria in the cytoplasm and mitochondria in the acid lysosome can be distinguished by mt-Keima. Briefly, Mt-Keima probe was transfected into cells at $37^{\circ} \mathrm{C}$ in $5 \% \mathrm{CO}_{2}{ }^{58}$. The fluorescence images were taken by Leica TCS SP5 II confocal spectroscopic microscope.

\section{Mitophagy flux}

Mitophagy flux was evaluated as a previous study described. In brief, MitoTracker Green FM $(500 \mathrm{nM})$ was used to stain cells. A CytoFlex platform (Beckman, South Kraemer Boulevard, Brea, CA, USA) was used for analysis. Mitophagy flux was defined as the ratio of MitoTracker Green FM fluorescence in the presence of mitophagy and lysosomal inhibitor (bafilomycin A1, Baf, $10 \mathrm{nM}$, SigmaAldrich, St. Louis, MO, USA) to that in the presence of only mitophagy.

\section{Determination of ATP levels}

An ATP Assay Kit (Beyotime Institute of Biotechnology) was applied for determination of the levels of ATP in HK-2 cells. After reperfusion for $6 \mathrm{~h}, \mathrm{HK}-2$ cells were harvested and then lysed in lysis buffer followed by centrifugation at $12,000 \times g$ for $10 \mathrm{~min}$ at $4^{\circ} \mathrm{C}$. ATP levels were then detected via mixing the supernatant $(50 \mathrm{~mL})$ with luciferase reagent $(50 \mathrm{~mL})$. As luciferase catalyzes the oxidation of luciferin to produce light via employing ATP, thus the emitted light is linearly associated with the levels of the ATP. Therefore, the ATP levels were evaluated with a microplate luminometer (Varioskan Flash, 5250040, Thermo).

\section{Reverse transcription qPCR}

Total RNA from kidney cortex and HK-2 cells was isolated with TRIzol reagent (Invitrogen). Then total RNA was reverse transcribed into cDNA with M-MLV Reverse Transcriptase (Invitrogen) and the miScript Reverse Transcription Kit (Qiagen, Germany) was used for miR145-5p reverse transcription. With SYBR Premix Ex Taq ${ }^{\mathrm{TM}}$ II (Takara, Dalian, China), RT-qPCR was performed on a 7500 Fast RT-qPCR System. The $2^{-\Delta \Delta C T}$ method was used to calculate the levels of MEG3, miR-145-5p, and RTKN, which were, respectively, normalized to U6 or glyceraldehyde 3-phosphate dehydrogenase (GAPDH). Primer sequences are listed in Supplementary Table.

\section{H\&E staining}

The kidney tissues of mice were harvested, fixed in formalin, embedded in paraffin, and dewaxed by xylene. Then kidney sections of $4 \mu \mathrm{m}$ were subjected to hematoxylin and rinsed by distilled water. Furthermore, the tissues were stained by eosin, hydrated by gradient ethanol, cleared by xylene, air-dried, and mounted by neutral balsam. The optical microscope (Olympus BX53, Japan) was utilized for the determination of the pathological change of kidney injury after staining. Histologic changes, including tubular necrosis, tubular detachment, and tubule vacuole, were examined in a blinded manner. Renal tubules with loss of brush border, tubular dilation and disruption, cast formation, and cell lysis were considered damaged.

\section{Luciferase reporter assay}

The wild-type or mutated sequence of MEG3 or RTKN was synthesized and subcloned into the pmirGLO-Dualluciferase reporter vector (Promega, Madison, MI, USA) to construct the pmirGLO-MEG3-WT (wild type of MEG3), pmirGLO-MEG3-Mut (mutant-type of MEG3), pmirGLO-RTKN-WT (wild type of RTKN), and pmirGLO-RTKN-Mut (mutant-type of RTKN) plasmids, respectively. Mutant form of MEG3 was used to destabilize the binding between Mut-MEG3 and miR-145-5p. The pmirGLO-MEG3-WT or pmirGLO-MEG3-Mut (pmirGLO-RTKN-WT or pmirGLO-RTKN-Mut) containing the predicted binding sites of miR-145-5p were cotransfected with $\mathrm{NC}$ mimics or miR-145-5p mimics into HK-2 cells using Lipofectamine 2000. The c-MYC target sequences within MEG3 promoter were subcloned into the pGL3-Basis vector for promoter analysis to construct the pGL3-MEG3 promoter plasmids. The plasmids were cotransfected with sh-c-MYC\#1/2 into the IR-treated HK-2 cells. After transfection for $48 \mathrm{~h}$, the relative luciferase activity was detected with a Dualluciferase Reporter Assay Kit (Promega), and the results were normalized to that of Renilla.

\section{Chromatin immunoprecipitation}

An EZ ChIP Chromatin Immunoprecipitation Kit (Millipore, Billerica, MA) was applied for ChIP assay. Chromatin was cross-linked in $1 \%$ formaldehyde for $10 \mathrm{~min}$ at $37^{\circ} \mathrm{C}$, sonicated to sizes of $200-1000 \mathrm{bp}$, followed by incubation with $3 \mu \mathrm{g}$ of magnetic beads coated with anti-c-MYC and anti-IgG antibody (Millipore) overnight at $4^{\circ} \mathrm{C}$ for $2 \mathrm{~h}$. Then protein/DNA complexes were eluted and reverse cross-linked. The immunoprecipitated DNA was analyzed by RT-qPCR.

\section{RNA immunoprecipitation}

RIP assays were conducted using the Magna RIP RNABinding Protein Immunoprecipitation Kit (Millipore, MA, 
USA). HK-2 cells were lysed with the RIPA lysis buffer. Then $100 \mu \mathrm{L}$ of cell extract was cultured with the RIPA buffer containing magnetic beads conjugated with human anti-Ago2 antibodies (Abcam, Cambridge, UK) or the IgG antibodies (Millipore, MA, USA). After antibody recovery by the protein $\mathrm{A} / \mathrm{G}$ beads, the relative enrichment of MEG3, miR-145-5p, and RTKN was detected by RT-qPCR.

\section{Western blot analysis}

RIPA lysate was used to extract proteins from renal tissues and HK-2 cells, and the concentrations were quantified by the Bicinchoninic Acid Kit (Pierce Biotechnology, Inc., Rockford, IL, USA). Sodium dodecyl sulfate-polyacrylamide gel electrophoresis was applied to isolate the proteins, and thereafter, separated proteins were transferred to polyvinylidene difluoride membranes (Millipore, Bradford, MA, USA). Membranes were blocked with 5\% skimmed milk powder for $2 \mathrm{~h}$ and then cultured with primary antibodies against Parkin (ab77924; Abcam, Cambridge, UK), p62 (ab56416; Abcam), LC3B (ab192870; Abcam), and GAPDH (ab181602; Abcam) at $4{ }^{\circ} \mathrm{C}$ overnight. Being washed twice with phosphate-buffered saline and Tween 20, the membranes were then cultured with horseradish peroxidase-labeled goat anti-rabbit secondary antibody for $1 \mathrm{~h}$ at $37^{\circ} \mathrm{C}$. Enhanced chemiluminescence reagent (Millipore; Merck KGaA) was used to visualize the bands. The densitometric analyses of western blots were subjected to ImageJ program.

\section{Serum biochemical measurement}

Serum creatinine and BUN levels in mice were measured by a commercially available clinical chemistry analyzer (Roche, Rotkreuz, Switzerland).

\section{Caspase-3 activity detection}

The caspase-3 activity in HK-2 cells was assessed via the Caspase-3 Colorimetric Assay Kit (Beyotime, Shanghai, China). HK-2 cells were lysed by the lysis buffer. Then the supernatant of the lysate was gathered, centrifuged, and cultured in Ac-DEVE-pNA and reaction buffer at $37^{\circ} \mathrm{C}$ for $2 \mathrm{~h}$. Finally, the optical density of caspase- 3 activity at $405 \mathrm{~nm}$ was measured by a microplate reader (BioTek, VT).

\section{TUNEL assay}

A TUENL assay was used to detect the TUNEL-positive cells in the kidney tissues. Briefly, tissue samples were fixed with formaldehyde and then dehydrated, paraffin embedded, and sectioned. Afterwards, the sections were dewaxed with xylene and hydrated with gradient ethanol. Thereafter, renal sections were cultured with $50 \mu \mathrm{L}$ of TUNEL reaction solution for $50 \mathrm{~min}$ followed by converting agent peroxidase for $30 \mathrm{~min}$ at $37^{\circ} \mathrm{C}$. Further, the sections were cultured with diaminobenzidine working solution for $10 \mathrm{~min}$, counterstained with hematoxylin for $3 \mathrm{~s}$, and sealed with neutral gum. Finally, the staining was observed under a microscope with five visual fields chosen from each group.

\section{Statistical analysis}

Statistical analyses were conducted using the GraphPad Prism 5 software. Data from at least three independent experiments are presented as the mean \pm standard deviation. Sample size was determined using G power for power analysis. All the data were normally distributed. Variance was similar between the groups that were being statistically compared. Student's $t$ test or one-way analysis of variance was performed to test differences between or among groups. $p<0.05$ was considered statistically significant.

\section{Author contributions}

D.L. and N.L. contributed to the performance of in vivo and in vitro IRI models, collection and assembly of data, and drafting of the manuscript. Y.L., X.Z., and N.L. contributed to data collection, analysis and interpretation, and manuscript revision. D.L. contributed to experimental design and revision of manuscript. Y.L. and N.L. were involved in experimental design, interpretation of data, critical revision of the manuscript, and final approval. All authors read and approved the final manuscript.

\section{Ethics statement}

The experimental protocol and animal use plan in this study were approved by the Animal Ethics Committee of Shengjing Hospital of China Medical University (Liaoning, China).

\section{Funding}

This work was supported by Department of Science \& Technology of Liaoning Province (No. 20170541057)

\section{Conflict of interest}

The authors declare no competing interests.

\section{Publisher's note}

Springer Nature remains neutral with regard to jurisdictional claims in published maps and institutional affiliations.

Supplementary information The online version contains supplementary material available at https://doi.org/10.1038/s41419-021-03466-5.

Received: 30 September 2020 Revised: 17 December 2020 Accepted: 19 January 2021

Published online: 18 February 2021

\footnotetext{
References

1. Yan, Y. et al. miR-214 represses mitofusin-2 to promote renal tubular apoptosis in ischemic acute kidney injury. Am. J. Physiol. Ren. Physiol. 318, F878-f87 (2020).

2. Malek, M. \& Nematbakhsh, M. Renal ischemia/reperfusion injury; from pathophysiology to treatment. J. Ren. Inj. Prev. 4, 20-27 (2015).

3. Snoeijs, M. G., van Heurn, L. W. \& Buurman, W. A. Biological modulation of renal ischemia-reperfusion injury. Curr. Opin. Organ Transplant. 15, 190-199 (2010).

4. Duann, P., Lianos, E. A., Ma, J. \& Lin, P. H. Autophagy, innate immunity and tissue repair in acute kidney injury. Int. J. Mol. Sci. 17, 662 (2016).

5. Ronco, C., Bellomo, R. \& Kellum, J. A. Acute kidney injury. Lancet 394 1949-1964 (2019).
} 
6. Kezic, A., Spasojevic, I., Lezaic, V. \& Bajcetic, M. Mitochondria-targeted antioxidants: future perspectives in kidney ischemia reperfusion injury. Oxid. Med. Cell. Longev. 2016, 2950503 (2016).

7. Sun, J. et al. Mitochondria in sepsis-induced AKI. J. Am. Soc. Nephrol. 30 1151-1161 (2019)

8. Gu, C. et al. Salidroside ameliorates mitochondria-dependent neurona apoptosis after spinal cord ischemia-reperfusion injury partially through inhibiting oxidative stress and promoting mitophagy. Oxid. Med. Cell. Longev. 2020, 3549704 (2020)

9. Wei, Q. \& Dong, Z. Mouse model of ischemic acute kidney injury: technical notes and tricks. Am. J. Physiol. Ren. Physiol. 303, F1487-F1494 (2012).

10. Valente, E. M. et al. Hereditary early-onset Parkinson's disease caused by mutations in PINK1. Science 304, 1158-1160 (2004).

11. Shao, D. et al. Increasing fatty acid oxidation prevents high-fat diet-induced cardiomyopathy through regulating parkin-mediated mitophagy. Circulation 142, 983-997 (2020).

12. Liu, S. et al. Autophagy plays a critical role in kidney tubule maintenance, aging and ischemia-reperfusion injury. Autophagy 8, 826-837 (2012).

13. Yang, X. et al. A network based method for analysis of IncRNA-disease associations and prediction of IncRNAs implicated in diseases. PLOS ONE 9, e87797 (2014).

14. Brandenburger, T., Salgado Somoza, A., Devaux, Y. \& Lorenzen, J. M. Noncoding RNAs in acute kidney injury. Kidney Int. 94, 870-881 (2018).

15. Salmena, L., Poliseno, L., Tay, Y., Kats, L. \& Pandolfi, P. P. A ceRNA hypothesis: the Rosetta Stone of a hidden RNA language? Cell 146 353-358 (2011)

16. Tang, B. et al. Downregulation of XIST ameliorates acute kidney injury by sponging miR-142-5p and targeting PDCD4. J. Cell. Physiol. 235, 8852-8863 (2020).

17. Cheng, W., Li, X. W., Xiao, Y. Q. \& Duan, S. B. Non-coding RNA-associated ceRNA networks in a new contrast-induced acute kidney injury rat model. Mol. Ther. Nucleic Acids 17, 102-112 (2019).

18. Tian, X. et al. LINC00520 targeting miR-27b-3p regulates OSMR expression level to promote acute kidney injury development through the PI3KAKT signaling pathway. J. Cell. Physiol. 234, 14221-14233 (2019).

19. Zhang, P. et al. The biomarker TCONS_00016233 drives septic AKI by targeting the miR-22-3p/AIFM1 signaling axis. Mol. Ther. Nucleic Acids 19, 1027-1042 (2020).

20. Alessio, E. et al. Single cell analysis reveals the involvement of the long noncoding RNA Pvt1 in the modulation of muscle atrophy and mitochondria network. Nucleic Acids Res. 47, 1653-1670 (2019).

21. Liu, C. et al. IncRNA RMST suppressed GBM cell mitophagy through enhancing FUS SUMOylation. Mol. Ther. Nucleic Acids 19, 1198-1208 (2020)

22. Liang, J., Wang, Q., Li, J. Q., Guo, T. \& Yu, D. Long non-coding RNA MEG3 promotes cerebral ischemia-reperfusion injury through increasing pyroptosis by targeting miR-485/AIM2 axis. Exp. Neurol. 325, 113139 (2020).

23. You, D. \& You, H. Repression of long non-coding RNA MEG3 restores nerve growth and alleviates neurological impairment after cerebral ischemiareperfusion injury in a rat model. Biomed. Pharmacother. 111, 1447-1457 (2019).

24. Huang, X., Gao, Y., Qin, J. \& Lu, S. The mechanism of long non-coding RNA MEG3 for hepatic ischemia-reperfusion: mediated by miR-34a/Nrf2 signaling pathway. J. Cell. Biochem. 119, 1163-1172 (2018).

25. Zou, L. et al. Long noncoding RNA-MEG3 contributes to myocardial ischemiareperfusion injury through suppression of miR-7-5p expression. Biosci. Rep. 39, BSR20190210 (2019).

26. Yan, $\mathrm{H}$. et al. Long non-coding RNA MEG3 functions as a competing endogenous RNA to regulate ischemic neuronal death by targeting miR-21/ PDCD4 signaling pathway. Cell Death Dis. 8, 3211 (2017).

27. Wei, G. H. \& Wang, X. IncRNA MEG3 inhibit proliferation and metastasis of gastric cancer via p53 signaling pathway. Eur. Rev. Med. Pharmacol. Sci. 21 3850-3856 (2017)

28. Wu, M. et al. LncRNA MEG3 inhibits the progression of prostate cancer by modulating miR-9-5p/QKI-5 axis. J. Cell. Mol. Med. 23, 29-38 (2019).

29. Zhang, X., Wu, N., Wang, J. \& Li, Z. LncRNA MEG3 inhibits cell proliferation and induces apoptosis in laryngeal cancer via miR-23a/APAF-1 axis. J. Cell. Mol. Med. 23, 6708-6719 (2019).

30. Fu, Z. J. et al. HIF-1a-BNIP3-mediated mitophagy in tubular cells protects against renal ischemia/reperfusion injury. Redox Biol. 36, 101671 (2020)
31. Tokgun, P. E., Tokgun, O., Kurt, S., Tomatir, A. G. \& Akca, H. MYC-driven regulation of long non-coding RNA profiles in breast cancer cells. Gene $\mathbf{7 1 4}$ 143955 (2019).

32. $\mathrm{Pu}$, Z . et al. LncRNA MEG3 contributes to adenosine-induced cytotoxicity in hepatoma HepG2 cells by downregulated ILF3 and autophagy inhibition via regulation PI3K-AKT-mTOR and beclin-1 signaling pathway. J. Cell. Biochem. 120, 18172-18185 (2019).

33. Xu, D. H., Chi, G. N., Zhao, C. H. \& Li, D. Y. Long noncoding RNA MEG3 inhibits proliferation and migration but induces autophagy by regulation of Sirt7 and PI3K/AKT/mTOR pathway in glioma cells. J. Cell. Biochem. https://doi.org/ 10.1002/jcb.28026 (2018).

34. Luo, H. C. et al. Role of long noncoding RNA MEG3/MiR-378/GRB2 axis in neuronal autophagy and neurological functional impairment in ischemic stroke. J. Biol. Chem. 295, 14125-14139 (2020).

35. Zhi, F. et al. Characteristic microRNA expression induced by $\delta$-opioid receptor activation in the rat liver under prolonged hypoxia. Cell. Physiol. Biochem. 44, 2296-2309 (2017)

36. Wu, G. et al miRNA-145-5p induces apoptosis after ischemia-reperfusion by targeting dual specificity phosphatase 6. J. Cell. Physiol. https://doi.org/10.1002/ jcp.28291 (2019)

37. Xie, $X$. et al. miR-145-5p/Nurr1/TNF-a signaling-induced microglia activation regulates neuron injury of acute cerebral ischemic/reperfusion in rats. Front. Mol. Neurosci. 10, 383 (2017).

38. Wei, B., Liu, Y. S. \& Guan, H. X. MicroRNA-145-5p attenuates high glucoseinduced apoptosis by targeting the Notch signaling pathway in podocytes. Exp. Ther. Med. 19, 1915-1924 (2020).

39. Dong, Y., Wei, M. H., Lu, J. G. \& Bi, C. Y. Long non-coding RNA HULC interacts with miR-613 to regulate colon cancer growth and metastasis through targeting RTKN. Biomed. Pharmacother. 109, 2035-2042 (2019).

40. Zhou, J. et al. MicroRNA-152 inhibits tumor cell growth by directly targeting RTKN in hepatocellular carcinoma. Oncol. Rep. 37, 1227-1234 (2017).

41. Zhang, W., Liang, Z. \& Li, J. Inhibition of rhotekin exhibits antitumor effects in lung cancer cells. Oncol. Rep. 35, 2529-2534 (2016).

42. Wang, S. et al. miR-145 inhibits breast cancer cell growth through RTKN. Int. J. Oncol. 34, 1461-1466 (2009).

43. Xiao, L. et al. Sustained activation of Wnt/ $\beta$-catenin signaling drives AKI to CKD progression. J. Am. Soc. Nephrol. 27, 1727-1740 (2016).

44. Li, Z. et al. (Pro)renin receptor is an amplifier of Wnt/ $\beta$-catenin signaling in kidney injury and fibrosis. J. Am. Soc. Nephrol. 28, 2393-2408 (2017).

45. Liu, X. J. et al. MicroRNA21 promotes interstitial fibrosis via targeting DDAH1: a potential role in renal fibrosis. Mol. Cell. Biochem. 411, 181-189 (2016).

46. $\mathrm{Li}, \mathrm{X}$. et al. Advanced oxidation protein products promote lipotoxicity and tubulointerstitial fibrosis via CD36/ $\beta$-catenin pathway in diabetic nephropathy. Antioxid. Redox Signal. 31, 521-538 (2019)

47. Wang, B. et al. Exosomal miR-1910-3p promotes proliferation, metastasis, and autophagy of breast cancer cells by targeting MTMR3 and activating the NFKB signaling pathway. Cancer Lett. 489, 87-99 (2020).

48. Wang, C. et al. FOXP1-induced IncRNA CLRN1-AS1 acts as a tumor suppressor in pituitary prolactinoma by repressing the autophagy via inactivating Wnt B-catenin signaling pathway. Cell Death Dis. 10, 499 (2019).

49. Fan, Q. et al. Autophagy promotes metastasis and glycolysis by upregulating MCT1 expression and Wnt/ß-catenin signaling pathway activation in hepatocellular carcinoma cells. J. Exp. Clin. Cancer Res. 37, 9 (2018).

50. Ruolan, W., Liangjiao, C. \& Longquan, S. The mTOR/ULK1 signaling pathway mediates the autophagy-promoting and osteogenic effects of dicalcium silicate nanoparticles. J. Nanobiotechnol. 18, 119 (2020).

51. Zhang, Y. Y. \& Feng, H. M. MEG3 suppresses human pancreatic neuroendocrine tumor cells growth and metastasis by down-regulation of mir-183. Cell. Physiol. Biochem. 44, 345-356 (2017).

52. Han, L., Dong, Z., Liu, N., Xie, F. \& Wang, N. Maternally expressed gene 3 (MEG3) enhances PC12 cell hypoxia injury by targeting miR-147. Cell. Physiol. Biochem. 43, 2457-2469 (2017)

53. Pang, $X$. et al. Knockdown of Rhotekin 2 expression suppresses proliferation and induces apoptosis in colon cancer cells. Oncol. Lett. 14, 8028-8034 (2017).

54. Xiang, G. et al. BNIP3L-dependent mitophagy accounts for mitochondrial clearance during 3 factors-induced somatic cell reprogramming. Autophagy 13, 1543-1555 (2017)

55. Xiong, J. et al. MYC is a positive regulator of choline metabolism and impedes mitophagy-dependent necroptosis in diffuse large B-cell lymphoma. Blood Cancer J. 7, e0 (2017) 
56. Montemurro, L. et al. A novel MYCN-specific antigene oligonucleotide deregulates mitochondria and inhibits tumor growth in MYCN-amplified neuroblastoma. Cancer Res. 79, 6166-6177 (2019).

57. Wang, J., Zhu, P., Li, R., Ren, J. \& Zhou, H. Fundc1-dependent mitophagy is obligatory to ischemic preconditioning-conferred renoprotection in ischemic
AKI via suppression of Drp1-mediated mitochondrial fission. Redox Biol. 30 101415 (2020).

58. Amanakis, G., Kleinbongard, P., Heusch, G. \& Skyschally, A. Attenuation of STsegment elevation after ischemic conditioning maneuvers reflects cardioprotection online. Basic Res. Cardiol. 114, 22 (2019). 